\title{
A Joint Physics-Based Statistical Deformable Model for Multimodal Brain Image Analysis
}

\author{
Christophoros Nikou, Associate Member, IEEE, Gloria Bueno, Fabrice Heitz*, and Jean-Paul Armspach
}

\begin{abstract}
A probabilistic deformable model for the representation of multiple brain structures is described. The statistically learned deformable model represents the relative location of different anatomical surfaces in brain magnetic resonance images (MRIs) and accommodates their significant variability across different individuals. The surfaces of each anatomical structure are parameterized by the amplitudes of the vibration modes of a deformable spherical mesh. For a given MRI in the training set, a vector containing the largest vibration modes describing the different deformable surfaces is created. This random vector is statistically constrained by retaining the most significant variation modes of its Karhunen-Loève expansion on the training population. By these means, the conjunction of surfaces are deformed according to the anatomical variability observed in the training set. Two applications of the joint probabilistic deformable model are presented: isolation of the brain from MRI using the probabilistic constraints embedded in the model and deformable model-based registration of three-dimensional multimodal (magnetic resonance/single photon emission computed tomography) brain images without removing nonbrain structures. The multiobject deformable model may be considered as a first step toward the development of a general purpose probabilistic anatomical atlas of the brain.
\end{abstract}

Index Terms-Brain isolation, image registration, magnetic resonance imaging (MRI), physically based deformable model, single photon emission computed tomography (SPECT), statistical shape models.

\footnotetext{
Manuscript received September 16, 1999; revised July 31, 2001. This work was supported by the "Groupement d'Intérêt Scientifique-Sciences de la Cognition" (CNRS, CEA, INRIA, MENESR). The work of C. Nikou was supported by the Commission of the European Communities, DG XII, in the framework of the Training and Mobility of Researchers (TMR) Program under Contract ERBFMIBCT960701. The Associate Editor responsible for coordinating the review of this paper and recommending its publication was J. Duncan. Asterisk indicates corresponding author.

C. Nikou was with the Université Louis Pasteur (Strasbourg I), Laboratoire des Sciences de l'Image, de l'Informatique et de la Télédétection, CNRS UPRES-A 7005 and the Institut de Physique Biologique, Faculté de Médecine, CNRS-UPRES-A 7004. He is now with the Aristotle University of Thessaloniki, Department of Informatics, Artificial Intelligence and Information Analysis Laboratory, 54006 Thessaloniki, Greece.

G. Bueno was with the Université Louis Pasteur (Strasbourg I), Laboratoire des Sciences de l'Image, de l'Informatique et de la Télédétection, CNRS UPRES-A 7005 and the Institut de Physique Biologique, Faculté de Médecine, CNRS-UPRES-A 7004. She is now with the Centro de Estudios e Investigaciones Tecnicas (CEIT) de Gipuzkoa, Paseo de Manuel Lardizabal 15, 20018 San Sebastian, Spain.

*F. Heitz is with the Université Louis Pasteur (Strasbourg I), Laboratoire des Sciences de l'Image, de l'Informatique et de la Télédétection, CNRS UPRES-A 7005, 4. Bd. Sébastien Brant, 67400 Illkirch, France (e-mail: Fabrice.Heitz@ensps.u-strasbg.fr).

J.-P. Armspach is with the Université Louis Pasteur (Strasbourg I) Institut de Physique Biologique, Faculté de Médecine, CNRS-UPRES-A 7004, 67085 Strasbourg, France.

Publisher Item Identifier S 0278-0062(01)09308-9.
}

\section{INTRODUCTION}

$\mathbf{I}$ $\mathrm{N}$ MEDICAL image analysis, deformable models offer a powerful approach to accommodate the significant variability of biological structures over time and across different individuals [1]. A survey on deformable models as a promising computer-assisted medical image analysis technique has recently been presented in [2]. Deformable models have principally been used to describe and characterize pathological shape deformations [3], [4], to register single modal images [5], [6], to label and segment different anatomical structures [7]-[9], or to track temporal structure deformations [10].

We present a three-dimensional (3-D) statistical deformable model (SDM) carrying information on multiple anatomical structures for multimodal brain image processing. The anatomical structures taken into consideration are head (skull and scalp), brain, ventricles, and cerebellum. Our goal is to describe the spatial relations between these anatomical structures as well as the biological shape variations observed over a representative population of individuals.

In the proposed approach, the surfaces of the anatomical structures of interest are first extracted from a training set of 3-D magnetic resonance imaging (MRI). To this end, a 24-patient training set is aligned in the same reference coordinate system and segmented using semi-automatic segmentation algorithms. Each segmented surface is parameterized by the amplitudes of the vibration modes of a physically based deformable model [10], [11] and a joint model is constructed for the different anatomical structures. The joint model is statistically constrained by a Karhunen-Loève (KL) decomposition of the vibration mode parameters. By these means, the spatial relation between the different structures, as well as the anatomical variability observed in the training set are compactly described by a limited number of parameters. The resulting joint SDM may be considered as a first step toward the development of a general purpose probabilistic atlas of the brain, for various applications in medical image analysis (segmentation, labeling, registration, and pathology characterization).

Two preliminary applications of the probabilistic deformable model are presented in this paper.

- The isolation of the brain [intracranial cavity (ICC)] from MRI using the probabilistic constraints embedded in the joint deformable model.

- The deformable model-based rigid registration of 3-D multimodal (MR/SPECT) brain images by optimizing an energy function relying on the chamfer distance between 
the statistically constrained model parts and the image data.

As related to our work, we have to mention the seminal research of Cootes et al. [12] on point distribution models (PDMs), which has strongly influenced the presented developments. In the PDM, two-dimensional shapes are described by the statistical distribution (over a training population) of selected boundary points. The statistical training of PDMs requires an exact point-to-point correspondence between the boundary points selected on different shapes. Manual point-to-point correspondence [12] becomes tedious or even unfeasible for 3-D shapes. Therefore, 3-D extensions of PDMs have been limited in practice to multislice models, but not to fully 3-D representations [7]. However, in the last two years, several techniques have been proposed to automate the extraction of features to be used as input to a PDM [13], [14].

Physics-based shape parameterizations [11], [10], [15] overcome these shortcomings by hierarchically describing a 3-D structure as the ordered superimposition of vibrations (of different frequencies) of an initial mesh. This approach is akin to the recent, independently published work of Kelemen et al. [8], who make use of a truncated hierarchical shape representation based on spherical harmonics. In contrast to standard PDM learning procedures, the shape statistics are calculated in the space of shape parameters rather than on point coordinates. To obtain a consistent learning of variability, all the shapes in the training set are first aligned in a reference coordinate system (the standard Talairach stereotactic system is for instance used in [8]). Small remaining misalignments (in translation and rotation) with respect to the reference coordinate system are learned with the statistical model. The model is, thus, robust to small misregistration in rotation and translation. The most prominent distinction from [8] is that in [8] the different cerebral structures are modeled and segmented independently, whereas in the proposed model, the different 3-D cerebral structures are parameterized in conjunction and included in a single (joint) statistical model. In the presented applications of the joint statistical model, the spatial and statistical relationships between the different model parts (skull and brain in particular) are strongly used to guide the segmentation and registration procedures.

The remainder of this paper is organized as follows: in Section II, the parameterization of the anatomical structures by the vibration modes of a spherical mesh is presented. The statistical training procedure is described in Section III. The applications of the probabilistic model to 3-D segmentation (brain isolation) and to multimodal (MRI/SPECT) image registration are presented in Section IV. This section includes experimental results on real data, with the 24-patients trained model. The performances of the model for 3-D image segmentation and multimodal volume registration have been assessed on 25 MRI/SPECT image pairs not belonging to the training set.

\section{ThreE-DimensionAl PhysiCs-BASED Deformable MODELING}

\section{A. Pre-Processing of the Data Base}

To provide a training set, a representative collection of 24 3-D MRI volumes of different patients have first been registered to a reference image using an unsupervised (robust) registration technique described in [16]. This initial registration step compensates for 3-D rotation, translation and scaling, which are not taken into account in the statistical model variability. All images are then referenced in the same coordinate system. As stated in [8], a major difficulty of application of statistical parametric models is the efficient establishment of correspondence between the different examples of the training set. A standard choice is the stereotactic reference coordinate system proposed by Talairach [8]. The use of this coordinate system requires the identification of the symmetry plane of the brain, as well as the position of the anterior and posterior commissures line (AC-PC), generally performed manually. To avoid tedious manual interaction on large data bases, we have resorted to automatic alignment procedures [16], which seem to provide a satisfactory alignment of the training volumes, as assessed by the experimental results. The same alignment is also applied to all the patient data processed in Section IV. We currently investigate other approaches for an automatic definition of the reference coordinate system and of the model parameterization to obtain an underlying Gaussian (zero mean) parameter distribution (as expected in the KL decomposition) [17].

Another issue concerns the cut-off of the bottom of the head. As can be seen on Fig. 4, this cut-off is highly variable, since it depends only on the position of the patient in the scan. At the present time, we have only a reduced number of 24 representative available examples in the training data base. The variability generated by head cut-off was, thus, not represented in the statistical model. All the training and patient data were, thus, normalized, by cutting all heads approximately at the same location.

The four considered anatomical structures (head, brain, ventricles, cerebellum) were then segmented, for each volume of the data base, using region-growing and semi-automatic techniques based on binary and grey-level morphological tools [18].

\section{B. Parameterization by Physics-Based Deformation Modes}

Each segmented anatomical structure was separately parameterized by the amplitudes of the vibration modes of a physics-based deformable model [10]. Following the approach of Nastar et al. [10], the model for a given structure consists of 3-D points sampled on a spherical surface, following a quadrilateral cylinder topology in order to avoid singularities due to the poles (we refer to [10] for details on this representation). Each node has a mass $m$ and is connected to its four neighbors with springs of stiffness $k$. The model nodes are stacked in vector

$$
\mathbf{X}_{0}=\left(x_{1}^{0}, y_{1}^{0}, z_{1}^{0}, \ldots, x_{N^{\prime} N}^{0}, y_{N^{\prime} N}^{0}, z_{N^{\prime} N}^{0}\right)^{T}
$$

where $N$ is the number of points in the direction of the geographical longitude and $N^{\prime}$ is the number of points in the direction of the geographical latitude of the sphere. The physical model is characterized by its mass matrix $\mathbf{M}$, its stiffness matrix $\mathbf{K}$ and its dumping matrix $\mathbf{C}$ and its governing equation may be written as [11]

$$
\mathbf{M} \ddot{\mathbf{U}}+\mathbf{C} \dot{\mathbf{U}}+\mathbf{K U}=\mathbf{F}
$$


where $\mathbf{U}$ stands for the nodal displacements of the initial mesh $\mathbf{X}_{0}$. The image force vector $\mathbf{F}$ is based on the Euclidean distance between the mesh nodes and their nearest contour points [19].

Since (2) is of order $3 N N^{\prime}$, where $N N^{\prime}$ is the total number of nodes of the spherical mesh, it is solved in a subspace corresponding to the $M_{s}$ truncated vibration modes of the deformable structure, $s$ [10], [11]. The number of vibration modes retained in the object description, was chosen so as to obtain a compact but adequately accurate representation. A typical a priori value covering many types of standard deformations is the quarter of the number of degrees of freedom (DOFs) in the system (i.e., $25 \%$ of the modes were kept). The number of DOFs of the original mesh, for all of the considered surfaces, $S=4$, was $S \times 3 N N^{\prime}=4 \times 3 \times 100 \times 100=120000$. In the vibration modes subspace, this number was then reduced to $3 \sum_{s=1}^{S}$ $M_{s}=3 \times 10000=30000$.

To solve (2) in the subspace corresponding to the truncated vibration modes the following change of basis is used:

$$
\mathbf{U}=\boldsymbol{\Phi} \tilde{\mathbf{U}}=\sum_{i} \tilde{u}_{i} \phi_{i}
$$

where

$\Phi \quad$ vector;

$\tilde{\mathbf{U}}$ matrix;

$\phi_{i} \quad i$ th column of $\boldsymbol{\Phi}$

$\tilde{u}_{i} \quad i$ th scalar component of vector $\tilde{\mathbf{U}}$.

By choosing $\boldsymbol{\Phi}$ as the matrix whose columns are the eigenvectors of the eigenproblem

$$
\mathbf{K} \phi_{i}=\omega_{i}^{2} \mathbf{M} \phi_{i}
$$

and using the standard Rayleigh hypothesis [10], matrices K, $\mathbf{M}$, and $\mathbf{C}$ are simultaneously diagonalized

$$
\left\{\begin{array}{l}
\boldsymbol{\Phi}^{T} \mathbf{M} \boldsymbol{\Phi}=\mathbf{I} \\
\boldsymbol{\Phi}^{T} \mathbf{K} \boldsymbol{\Phi}=\mathbf{\Omega}^{2}
\end{array}\right.
$$

where $\Omega^{2}$ is the diagonal matrix whose elements are the eigenvalues $\omega_{i}^{2}$ and $\mathbf{I}$ is the identity matrix.

An important advantage of this formulation is that the eigenvectors and the eigenvalues of a quadrilateral mesh with cylinder topology have an explicit expression [20] and they do not have to be computed by slower eigendecomposition techniques (generally matrices $\mathbf{K}$ and $\mathbf{M}$ are very large). The eigenvalues are given by

$$
\omega_{p, p^{\prime}}^{2}=\frac{4 k}{m}\left(\sin ^{2} \frac{p \pi}{2 N}+\sin ^{2} \frac{p^{\prime} \pi}{N^{\prime}}\right)
$$

and the eigenvectors are obtained by

$$
\phi_{p, p^{\prime}}=\left[\ldots, \cos \frac{(2 n-1) p \pi}{2 N} \cos \frac{2 n^{\prime} p^{\prime} \pi}{N^{\prime}}, \ldots\right]^{T}
$$

with $n \in\{1,2, \ldots, N\}$ and $n^{\prime} \in\left\{1,2, \ldots, N^{\prime}\right\}$.

Substituting (3) into (2) and premultiplying by $\boldsymbol{\Phi}^{T}$ yields

$$
\ddot{\tilde{\mathbf{U}}}+\tilde{\mathbf{C}} \dot{\tilde{\mathbf{U}}}+\boldsymbol{\Omega}^{2} \tilde{\mathbf{U}}=\tilde{\mathbf{F}}
$$

In many computer vision applications [11], when the initial and the final state are known, it is assumed that a constant load $\mathbf{F}$ is applied to the body. Thus, (2) is called the equilibrium governing equation and corresponds to the static problem

$$
\mathbf{K U}=\mathbf{F} .
$$

In the new basis, (9) is, thus, simplified to $3 N N^{\prime}$ scalar equations

$$
\omega_{i}^{2} \tilde{u}_{i}=\tilde{f}_{i} .
$$

In (10), $\omega_{i}$ designates the $i$ th eigenvalue, the scalar $\tilde{u}_{i}$ is the amplitude of the corresponding vibration mode (corresponding to eigenvector $\phi_{i}$ ). Equation (10), indicates that instead of computing the displacements vector $\mathbf{U}$ from (9), we can compute its decomposition in terms of the vibration modes of the original mesh.

Fig. 1 shows the vibration modes based parameterization of the four different anatomical surfaces considered here, for a subject belonging to the training set. The $25 \%$ lowest frequency modes were retained for this representation. Although not providing a high-resolution description of the surfaces, this truncated representation provides a satisfactory compromise between accuracy and complexity of the representation. The spherical model is initialized around the structures of interest [Fig. 1(a), (d), (g), and (j)]. The vibration amplitudes are explicitly computed by (10), where rigid body modes $\left(\omega_{i}=0\right)$ are discarded and the nodal displacements may be recovered using (3). The physical representation $\mathbf{X}(\tilde{\mathbf{U}})$ is finally given by applying the deformations to the initial spherical mesh [Fig. 1(b)-(c), (e)-(f), (h)-(i), and (k)-(l)]

$$
\mathbf{X}(\tilde{\mathbf{U}})=\mathbf{X}_{0}+\boldsymbol{\Phi} \tilde{\mathbf{U}} \text {. }
$$

Thus, the different surfaces of a particular patient are hierarchically described in terms of vibrations of an initial spherical mesh. The next step consists in applying the above parameterization to each patient of the training set and to perform statistical learning for the anatomical structures taken into consideration.

\section{Statistical Training: The Joint Model}

For each image $i=1, \ldots, n(n=24)$ in the training set, a vector $\mathbf{a}_{i}$ containing the lowest frequency vibration modes describing the different $S=4$ anatomical structures is created

$$
\mathbf{a}_{i}=\left(\tilde{\mathbf{U}}_{i}^{1}, \tilde{\mathbf{U}}_{i}^{2} \ldots, \tilde{\mathbf{U}}_{i}^{S}\right)^{T}
$$

where

$$
\tilde{\mathbf{U}}_{i}^{s}=\left(\tilde{u}_{1}^{s}, \tilde{u}_{2}^{s}, \ldots, \tilde{u}_{M_{s}}^{s}\right)_{i}
$$

with $3 \sum_{s=1}^{S} M_{s}<12 N N^{\prime}$. In the joint statistical model constructed here, the first structure $(s=1)$ corresponds to the head, the second to the brain, the third to the ventricles and the last one $(s=4)$ to the cerebellum.

Random vector $\mathbf{a}$ is statistically constrained by retaining the most significant variation modes in its KL transform [12], [21], [15]

$$
\mathbf{a}=\overline{\mathbf{a}}+\mathbf{P b}
$$

where $\tilde{\mathbf{C}}=\boldsymbol{\Phi}^{T} \mathbf{C} \boldsymbol{\Phi}$ and $\tilde{\mathbf{F}}=\boldsymbol{\Phi}^{T} \mathbf{F}$. 


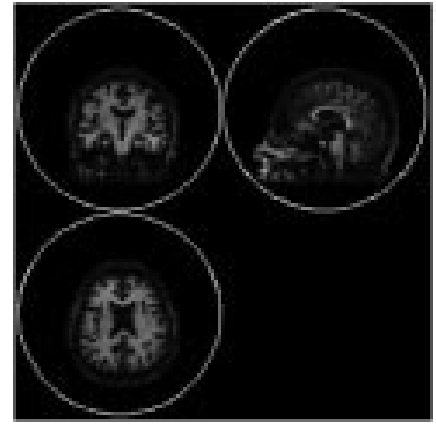

(a)

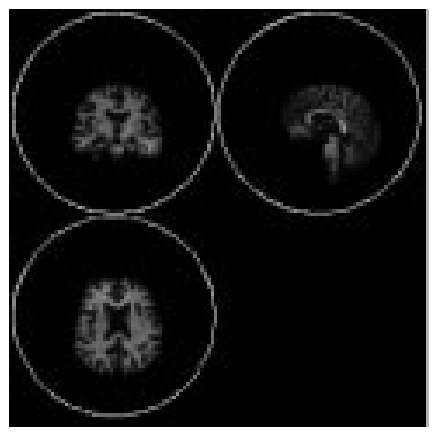

(d)

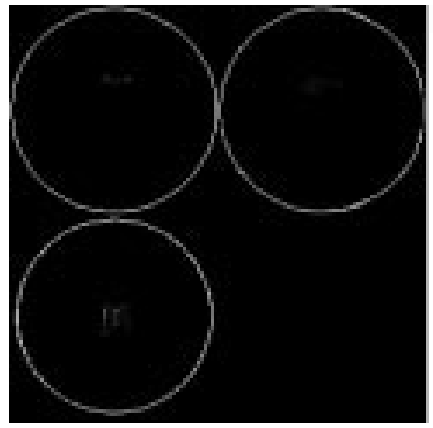

(g)

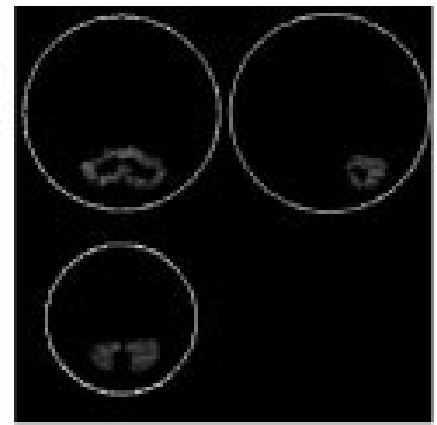

(j)

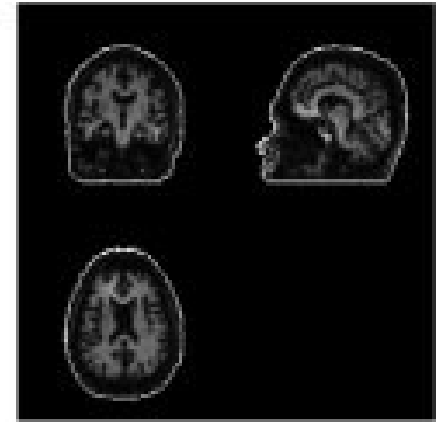

(b)

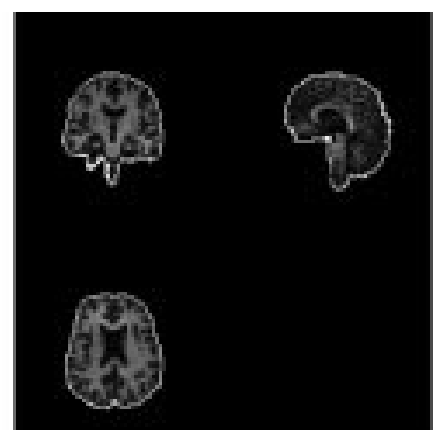

(e)

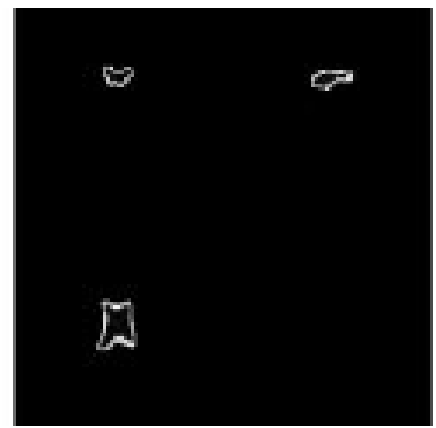

(h)

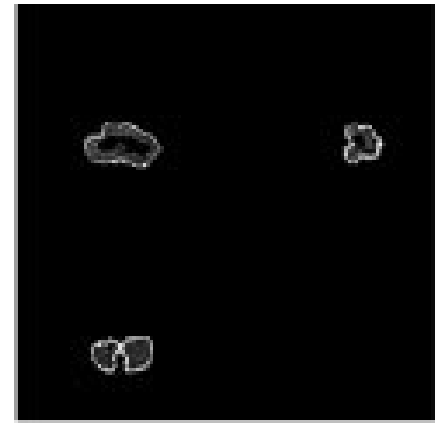

(k)

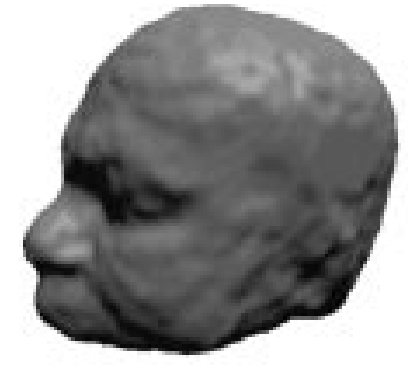

(c)

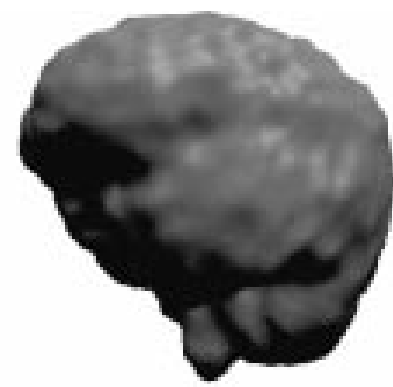

(f)

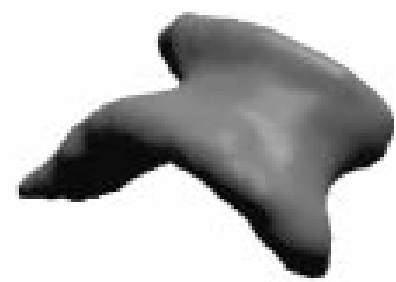

(i)

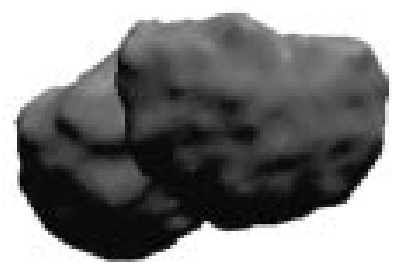

(1)

Fig. 1. Parameterization of the different anatomical structures from 3-D MRI. The first column shows the initial spherical mesh superimposed on the structures to be parameterized. Structures are shown in a multiplanar (sagittal, coronal, transversal) view. The middle column presents a multiplanar view of the deformable models at equilibrium (25\% of the vibration modes are kept). The last column shows 3-D renderings of the physically based models. The rows from top to bottom correspond to: (a)-(c) head, (d)-(f) brain, (g)-(i) ventricles and (j)-(l) cerebellum.

where

$$
\overline{\mathbf{a}}=\frac{1}{n} \sum_{i=1}^{n} \mathbf{a}_{i}
$$

is the average vector of vibration amplitudes of the structures belonging to the training set, $\mathbf{P}$ is the matrix whose columns are the eigenvectors of the covariance matrix

$$
\boldsymbol{\Gamma}=\mathbb{E}\left[(\mathbf{a}-\overline{\mathbf{a}})^{T}(\mathbf{a}-\overline{\mathbf{a}})\right]
$$

and

$$
\mathbf{b}_{i}=\mathbf{P}^{T}\left(\mathbf{a}_{i}-\overline{\mathbf{a}}\right)
$$

are the coordinates of $(\mathbf{a}-\overline{\mathbf{a}})$ in the eigenvector basis. 


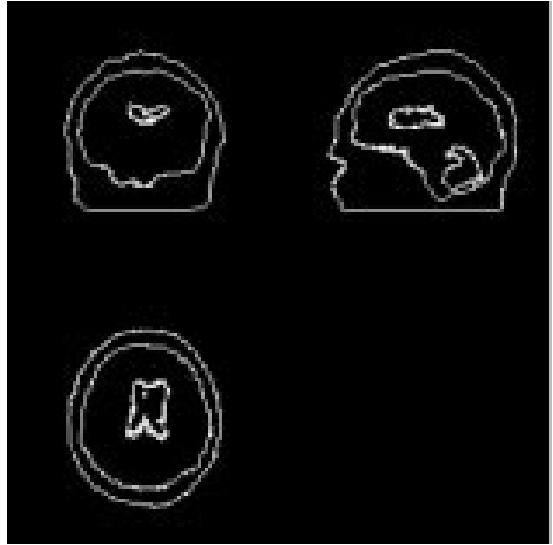

(a)

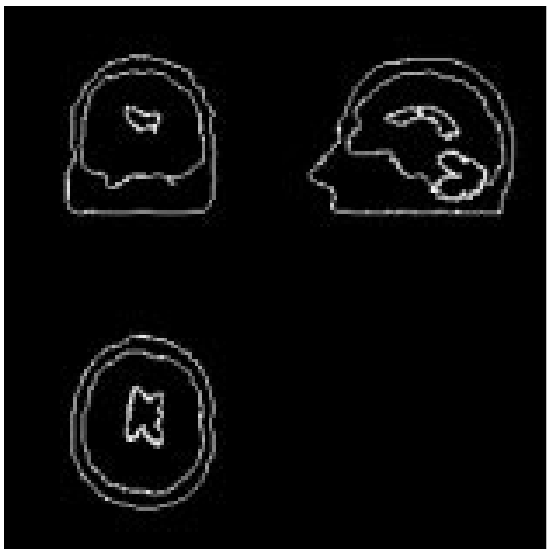

(d)

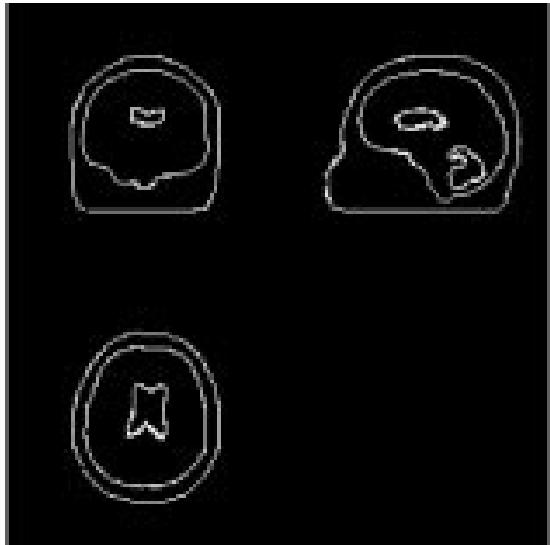

(b)

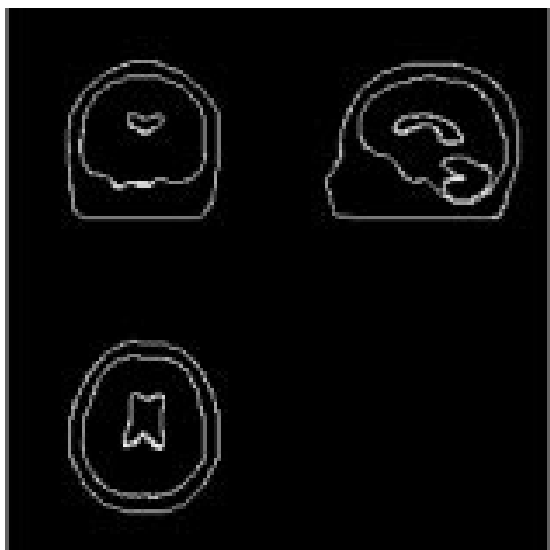

(e)

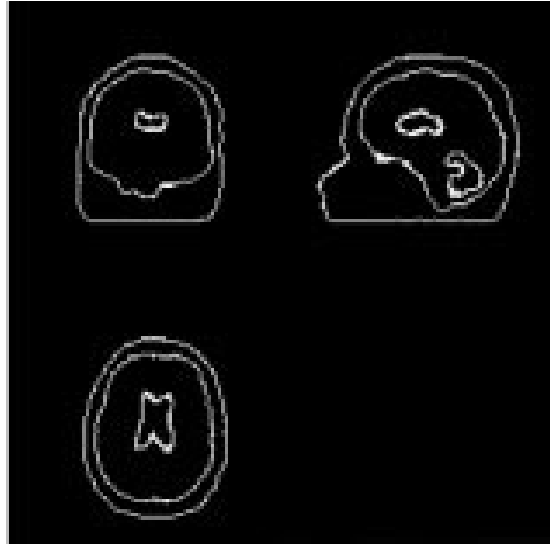

(c)

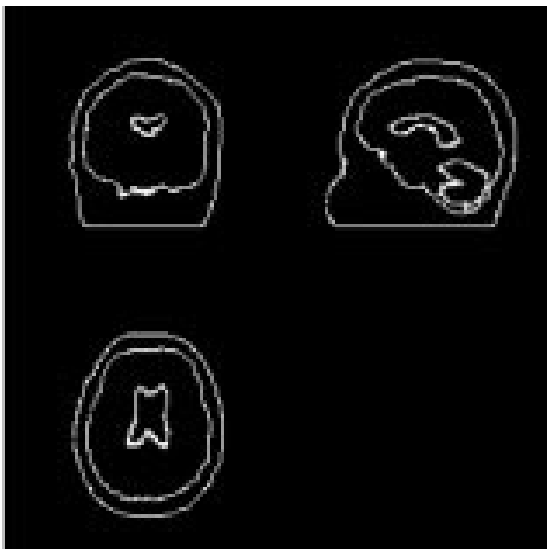

(f)

Fig. 2. Deformations of the 3-D joint model by varying the first two statistical modes in vector $\mathbf{b}$ between $-\sqrt{\lambda_{i}}$ and $\sqrt{\lambda_{i}}, i=1,2$. $\lambda_{i}$ designates the $i$ th eigenvalue of the covariance matrix $\boldsymbol{\Gamma}$. Each image shows a multiplanar (sagittal, coronal, transversal) view of the $3-\mathrm{D}$ model. (a) $\mathbf{b}[1]=-3 \sqrt{\lambda_{1}}$. (b) $\mathbf{b}[1]=0$. (c) $\mathbf{b}[1]=3 \sqrt{\lambda_{1}}$. (d) $\mathbf{b}[2]=-3 \sqrt{\lambda_{2}}$. (e) $\mathbf{b}[2]=0$. (f) $\mathbf{b}[2]=3 \sqrt{\lambda_{2}}$.

The joint deformable model is finally parameterized by the $m$ most significant statistical deformation modes stacked in vector $\mathbf{b}$. By modifying $\mathbf{b}$, the set of anatomical structures is deformed in conjunction (Fig. 2), according to the anatomical variability observed in the training set. The multiobject deformable model describes the spatial relationships between the considered surfaces of a subject as well as their shape variations.

Given a set of $S=4$ initial spherical meshes, corresponding to the number of structures described by the joint model

$$
\mathbf{X}_{I N I T}=\left(\begin{array}{c}
\mathbf{X}_{0}^{1} \\
\vdots \\
\mathbf{X}_{0}^{S}
\end{array}\right)
$$

the SDM X(a) is, thus, represented by

$$
\mathbf{X}(\mathbf{a})=\mathbf{X}_{I N I T}+\underline{\overline{\mathbf{\Phi}}} \mathbf{a} .
$$

Combining (14) and (19), we have

$$
\mathrm{X}(\mathrm{b})=\mathrm{X}_{I N I T}+\underline{\overline{\mathbf{\Phi}}} \overline{\mathbf{a}}+\underline{\overline{\mathbf{\Phi}}} \mathbf{P b}
$$

where

$$
\begin{aligned}
& \underline{\mathbf{\Phi}}=\left(\begin{array}{cccc}
\boldsymbol{\Phi}_{1} & \mathbf{0} & \cdots & \mathbf{0} \\
\mathbf{0} & \mathbf{\Phi}_{2} & \cdots & \mathbf{0} \\
\vdots & \vdots & \ddots & \vdots \\
\mathbf{0} & \mathbf{0} & \cdots & \boldsymbol{\Phi}_{S}
\end{array}\right) \\
& \mathbf{P}=\left(\begin{array}{c}
\mathbf{P}_{1} \\
\vdots \\
\mathbf{P}_{S}
\end{array}\right), \quad \overline{\mathbf{a}}=\left(\begin{array}{c}
\overline{\mathbf{a}}_{1} \\
\vdots \\
\overline{\mathbf{a}}_{S}
\end{array}\right) .
\end{aligned}
$$

In (21) and (22), the columns of any $3 N N^{\prime} \times 3 M_{s}$ matrix $\boldsymbol{\Phi}_{s}$ are the eigenvectors of the spherical mesh describing surface $s$. Each $3 M_{s} \times m$ matrix $\mathbf{P}_{s}$ describes also the statistical dependencies between the structures deformations observed in the training set. Each vector $\overline{\mathbf{a}}_{s}$ is of order $3 M_{s} \times 1$ and vector $\mathrm{b}$ has a low dimension $m \ll 3 \sum_{s=1}^{S} M_{s}$.

As can be seen in Table I, with the KL representation, only a few parameters are necessary to describe the variations in the training population (Fig. 2). For instance, seven parameters carry approximately $95 \%$ of the global information. In practice, we keep $m \simeq 10$ parameters for this representation (another criterion for ordering the deformation modes, based on maximal local deformations is proposed in [8]). 
TABLE I

Percentage of the Global InFormation CARried by the DifFerent Eigenvalues Associated With the Statistical Model. The Number of NONZERO EIGENVALUES IS 24

\begin{tabular}{c|c|c}
\hline & & $\sum_{k}$ \\
$\lambda_{k}$ & $\frac{\sum_{i=1}^{k} \lambda_{i}}{\sum_{i=1}^{24} \lambda_{i}}(\%)$ & $\sum_{i=1}^{24} \lambda_{i}$ \\
& & \\
\hline \hline$\lambda_{1}$ & 47,22 & 47.22 \\
$\lambda_{2}$ & 14.96 & 62.18 \\
$\lambda_{3}$ & 9.26 & 71.44 \\
$\lambda_{4}$ & 9.09 & 80.53 \\
$\lambda_{5}$ & 6.39 & 86.92 \\
$\lambda_{6}$ & 4.69 & 91.61 \\
$\lambda_{7}$ & 3.86 & 95.47 \\
$\vdots$ & $\vdots$ & $\vdots$ \\
$\lambda_{25}$ & 0.00 & 100.0 \\
\hline
\end{tabular}

Finally in the KL subspace the DOFs are reduced to $m \simeq 10$ achieving a compression ratio of $12000: 1$. This compression ratio enables a compact description of shape variability, and results in a tractable constrained deformable model for brain image segmentation and registration, as described in Section IV.

\section{Application to 3-D SEgmentation AND REGiSTRATION}

Several applications of the statistical model may be considered in brain image processing. The model may be used as a simplified anatomical representation of the images belonging to the training set. If the training set is representative enough of a population, the model may also be used to analyze images of patients not belonging to the training set. To this end, the 24 subjects of our data base were carefully selected, with the aid of an expert neurologist. Additionally, the data base is conceived in such a way that it can be incrementally augmented by new elements.

We describe here two applications of the joint statistical model: the isolation of the brain (the ICC) from 3-D MRI and the registration of multimodal (MRI/SPECT) brain images.

Before presenting these two applications, let us notice that the equation describing the configuration of the statistical model

$$
\mathbf{X}(\mathbf{b})=\mathbf{X}_{I N I T}+\underline{\overline{\mathbf{\Phi}}} \overline{\mathbf{a}}+\underline{\mathbf{\Phi}} \mathbf{P b}
$$

may be separated into $S$ coupled equations describing the different anatomical parts of the model

$$
\begin{array}{ccc}
\mathbf{X}_{1}(\mathbf{b}) & = & \mathbf{X}_{0}^{1}+\boldsymbol{\Phi}_{1} \overline{\mathbf{a}}_{1}+\boldsymbol{\Phi}_{1} \mathbf{P}_{1} \mathbf{b} \\
\vdots & \vdots & \vdots \\
\mathbf{X}_{S}(\mathbf{b}) & = & \mathbf{X}_{0}^{S}+\boldsymbol{\Phi}_{S} \overline{\mathbf{a}}_{S}+\boldsymbol{\Phi}_{S} \mathbf{P}_{S} \mathbf{b} .
\end{array}
$$

\section{A. Deformable Model-Based Segmentation}

In this section, we present a first application of the statistical model, corresponding to the segmentation of the ICC, also called brain isolation.

In order to segment the ICC from a patient MRI volume, not belonging to the training set, the patient head is first parameterized by the physics-based model. The head structure is easily segmented from its background by simple thresholding and region growing algorithms. The segmented head surface is parameterized by the amplitudes of the vibration modes of a spherical mesh, as already explained in Section II. The spherical mesh is initialized around the head structure and (2) is solved in the modal subspace. Let us recall that for the formulation used below, the head structure is considered as the first structure described by the model $(s=1)$. According to (10), the solution for the vibration amplitudes describing the patient head surface is

$$
\tilde{u}_{i}^{1}=\frac{1}{\omega_{i}^{2}} \tilde{f}_{i}^{1}
$$

for $i=1, \ldots, 3 M_{1}$. The head surface coordinates are obtained by introducing vector $\tilde{\mathbf{U}}^{1}=\left(\widetilde{u}_{1}^{1}, \widetilde{u}_{2}^{1}, \ldots, \widetilde{u}_{M_{1}}^{1}\right)^{T}$ in (11)

$$
\mathbf{X}_{1}\left(\tilde{\mathbf{U}}^{1}\right)=\mathbf{X}_{0}^{1}+\boldsymbol{\Phi}_{1} \tilde{\mathbf{U}}^{1} .
$$

The next step consists in determining the statistical model parameters $\mathbf{b}$ that best describe the segmented head surface

$$
\mathbf{X}_{1}(\mathbf{b})=\mathbf{X}_{0}^{1}+\boldsymbol{\Phi}_{1} \overline{\mathbf{a}}_{1}+\boldsymbol{\Phi}_{1} \mathbf{P}_{1} \mathbf{b}=\mathbf{X}_{1}\left(\tilde{\mathbf{U}}^{1}\right) .
$$

System (27) is overconstrained: there are $3 N N^{\prime}$ equations (the head surface coordinates $\mathbf{X}_{1}$ ) and $m$ unknowns (the components of $b$ ). The overconstrained system is solved by standard least-squares, yielding the following pseudoinverse solution for the deformation parameter $b$

$$
\begin{aligned}
\mathbf{b}^{*}= & {\left[\left(\boldsymbol{\Phi}_{1} \mathbf{P}_{1}\right)^{T} \boldsymbol{\Phi}_{1} \mathbf{P}_{1}\right]^{-1}\left(\boldsymbol{\Phi}_{1} \mathbf{P}_{1}\right)^{T} } \\
& \cdot\left[\mathbf{X}_{1}\left(\tilde{\mathbf{U}}^{1}\right)-\mathbf{X}_{0}^{1}-\boldsymbol{\Phi}_{1} \overline{\mathbf{a}}_{1}\right] .
\end{aligned}
$$

The other patient anatomical structures surfaces (brain, etc.) are then recovered by introducing the estimated parameter $\mathrm{b}^{*}$ in (24), describing the other parts $(s=2,3,4)$ of the statistical model

$$
\begin{array}{cccc}
\mathbf{X}_{2}\left(\mathbf{b}^{*}\right) & = & \mathbf{X}_{0}^{2}+\boldsymbol{\Phi}_{2} \overline{\mathbf{a}}_{2}+\boldsymbol{\Phi}_{2} \mathbf{P}_{2} \mathbf{b}^{*} \\
\vdots & \vdots & \vdots \\
\mathbf{X}_{S}\left(\mathbf{b}^{*}\right) & = & \mathbf{X}_{0}^{S}+\boldsymbol{\Phi}_{S} \overline{\mathbf{a}}_{S}+\boldsymbol{\Phi}_{S} \mathbf{P}_{S} \mathbf{b}^{*} .
\end{array}
$$

Equation (29) provides a prediction of the location of the considered surfaces, obtained by exploiting the spatial relationships between the head and the other anatomical structures, coded in the learned statistical representation. This initial prediction may be further refined by standard iterative refinement algorithms such as energy-based approaches [22], iterative closest point techniques [23] or grey-level profile matching [8]. This refinement step has not been considered here, to separately evaluate the contribution of the statistical information embedded in the learned statistical representation.

To summarize, the overall segmentation algorithm is based on the following steps.

1) Parameterization of the head surface using (25) and (26).

2) Estimation of the statistical deformation parameters $\mathbf{b}^{*}$ by solving the regularized overconstrained system (28).

3) Prediction of the brain (ICC) surface by (29).

4) Eventually iterative refinement of the solution to fit more precisely the data grey-level profile. 


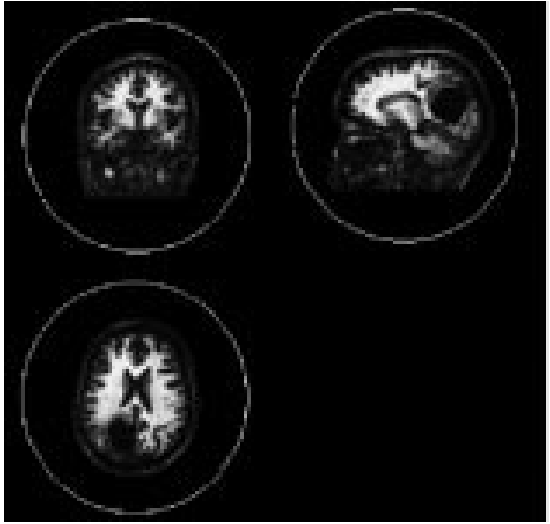

(a)

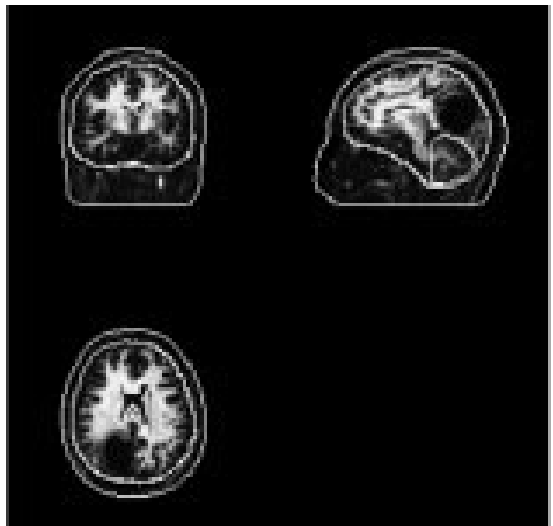

(b)

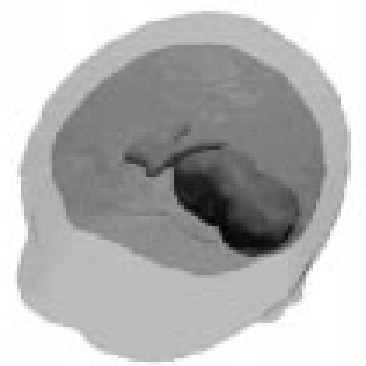

(c)

Fig. 3. Segmentation of a postoperative MRI. (a) Patient MR image with the initial spherical mesh superimposed. (b) Prediction of the different anatomical structures surfaces using the head surface in (a) and the probabilistic deformable model. (c) Three-dimensional rendering of (b).

Steps 1)-3) take about 5 min cpu time on a standard (HP 9000/C200) workstation for a $128^{3}$ image volume.

\section{B. Results and Validation of Segmentation}

This section shows some results of ICC segmentation, on our 3-D database. Fig. 3 presents a typical example of the segmentation from a 3-D MRI, corresponding to a patient not belonging to the training set. The image in Fig. 3(a) is a postoperative MRI (exhibiting missing data). In Fig. 3(b), the head surface is segmented and parameterized by the physics-based deformable model [eqs. (25) and (26)]. In Fig. 3(c), the head surface coordinates combined with the probabilistic model provide a prediction for the other anatomical structures. The statistical model is not affected by missing data because its deformations are constrained by the statistical analysis of the shape variations observed in the training population.

Experiments show that the initial prediction of the internal brain structures given by the model requires further refinement in order to obtain an accurate segmentation (this could be the topic of a future work). However, the results concerning brain (ICC) isolation were good enough to take them into consideration, without further improvement of the initial solution.

ICC segmentations results may be qualitatively evaluated in Fig. 4, on 12 typical MRIs not belonging to the training data set. The estimated brain contours are superimposed onto the original 3-D MRIs and the corresponding 3-D surfaces are displayed. Quantitative evaluation was carried out on 25 MRIs not belonging to the training data base. The automatic ICC segmentation obtained with the deformable model was compared with the results given by expert manual segmentation (considered as ground truth) using region growing and semi-automatic techniques based on binary and grey-level morphological tools [18].

Several measures have been suggested, to assess the performances of segmentation algorithms [8], [24]. Let $G T$ (ground truth), denote the segmented volume obtained by human experts, $G T^{c}$ its complement, $S e g$ the isolated brain obtained by the SDM-based approach and $|E|$ the number of elements (voxels) of a set $E$. The following complementary quality measures have been considered.
- Measures inspired by receiver operating characteristics in statistical detection theory [24], [25]:

- the sensitivity (also called true positives fraction), corresponding to the probability of detection defined here by

$$
\text { sensitivity }=\frac{|\operatorname{Seg} \cap G T|}{|G T|}
$$

- the specificity, defined as $\{1-$ false positives fraction $\}$ corresponding to $\{1-$ probability of false alarm\}

$$
\text { specificity }=1-\frac{|S e g-G T|}{\left|G T^{c}\right|}
$$

- the accuracy defined as the fraction of object and background voxels which are segmented properly. This measure is related to the previous ones by: accuracy $=\{$ sensitivity $*$ actual positives fraction + specificity $*$ actual negatives fraction $\}$, and may be computed by

$$
\text { accuracy }=\frac{|G T \cap \operatorname{Seg}|+\left|G T^{c}-(\operatorname{Seg}-G T)\right|}{\left|G T \cup G T^{c}\right|} .
$$

- An overlap measure, proposed in [8], defined as

$$
\text { overlap }=\frac{|G T \cap \operatorname{Seg}|}{|G T \cup \operatorname{Seg}|} .
$$

The overlap measure is also considered as a strong test for segmentation accuracy, since for example two voxel cubes of a volume of $10 \times 10 \times 10$, shifted by one voxel along the space diagonal direction results in only $57 \%$ overlap [8].

Table II shows the mean and the standard deviation of these different quality measures, for the 25 processed MRIs. As can be seen, the expert segmentation and the automatic brain isolation are in good agreement, for all quality measures. The initial prediction, provided by the SDM is close to a semi-manual expert segmentation, without any further refinement. 

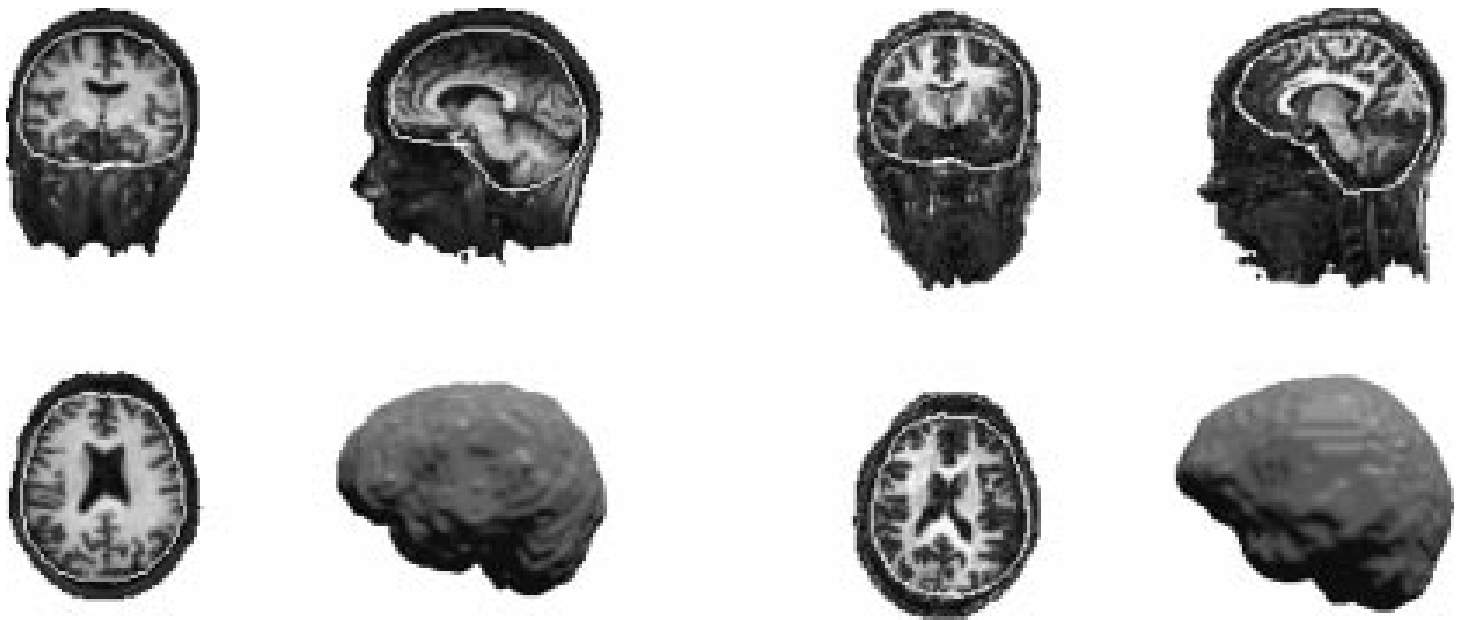

(a)
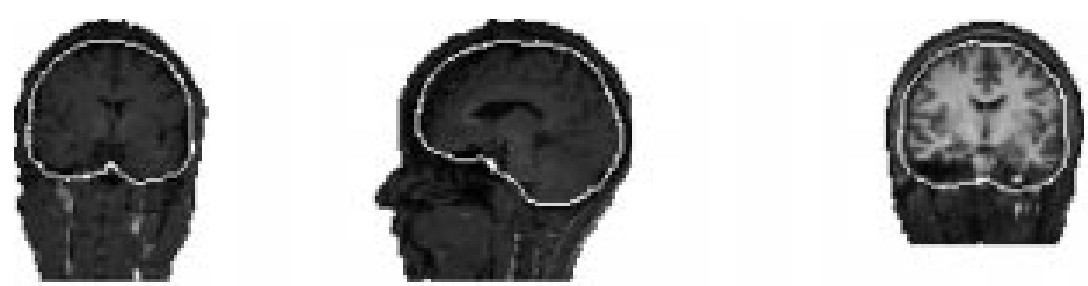

(b)
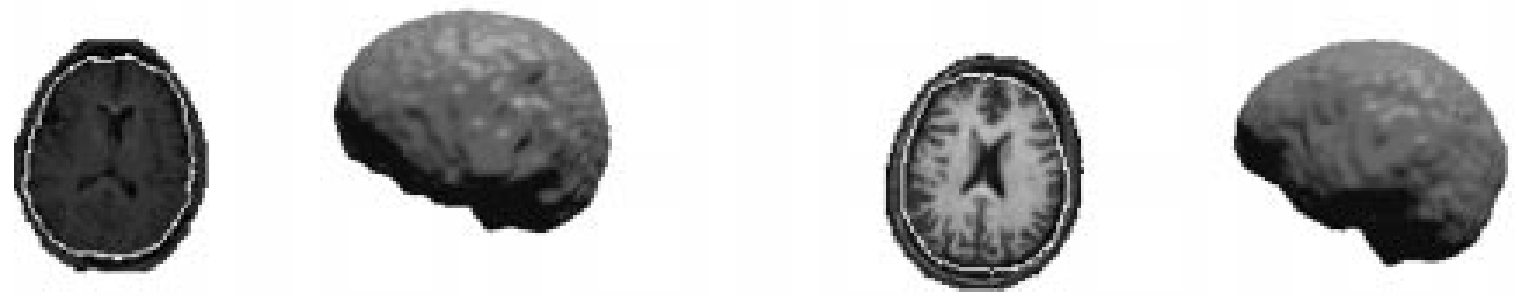

(c)

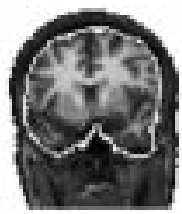

(d)
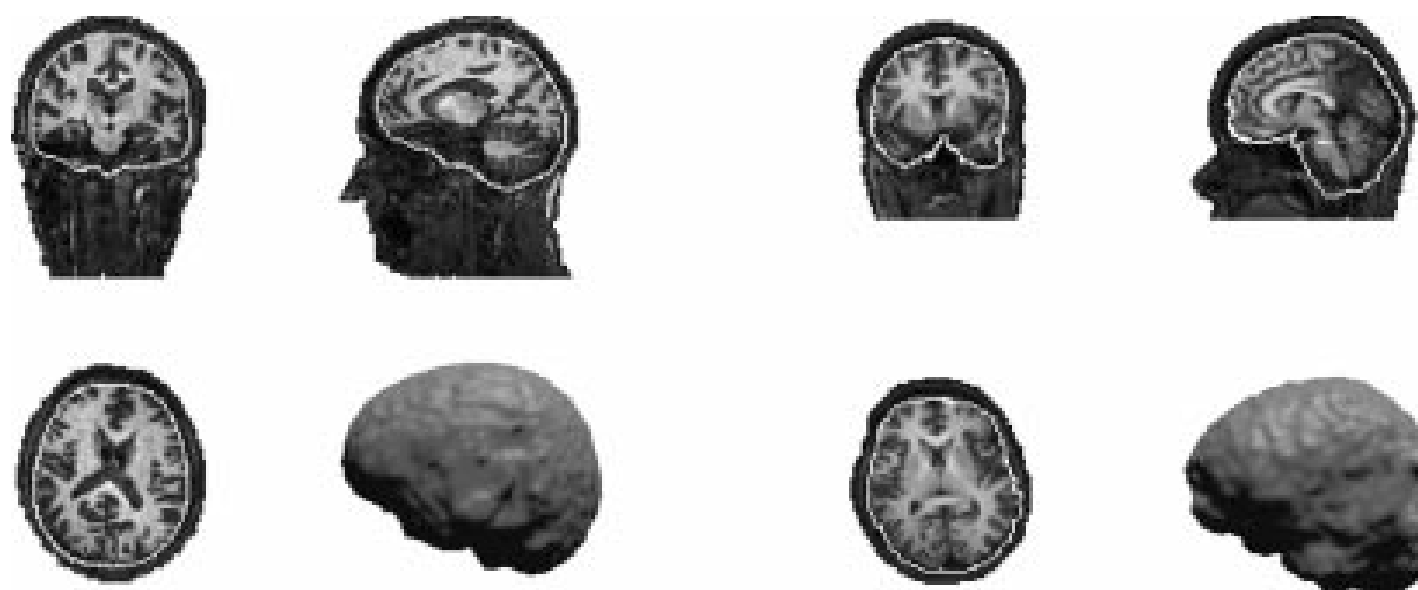

(e)
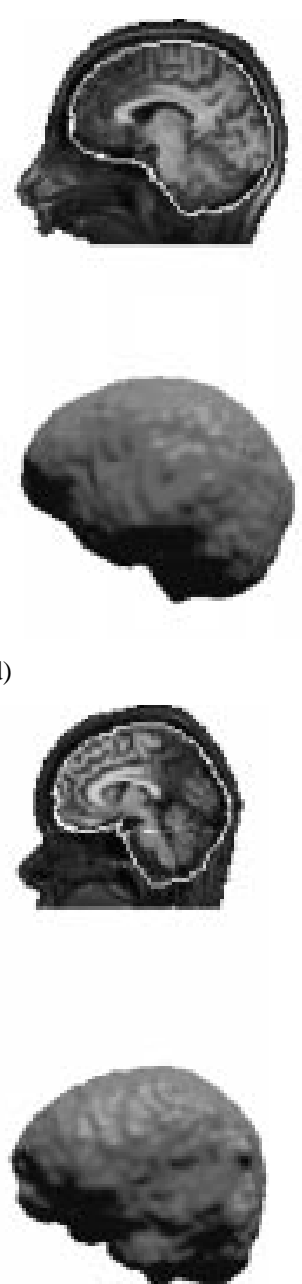

(f)

Fig. 4. (a)-(f) Brain isolation on 3-D MRIs. The multiplanar view of the isolated brain is superimposed on the original MRI data. A 3-D rendering of the corresponding surface is also displayed.

\section{Multimodal Image Registration}

The second application concerns the rigid registration of 3-D multimodal (MR/SPECT) images. Registration of a multimodal image pair consists in estimating the rigid transformation parameters (3-D rotation and translation parameters) that have to be applied to the image to be registered (here the SPECT image) in order to match the reference image (here, the MRI).

The registration relies on the head structure in the MRI and the brain surface in the SPECT image, which are easy to extract from these two modalities (in contrast to the brain structure in 

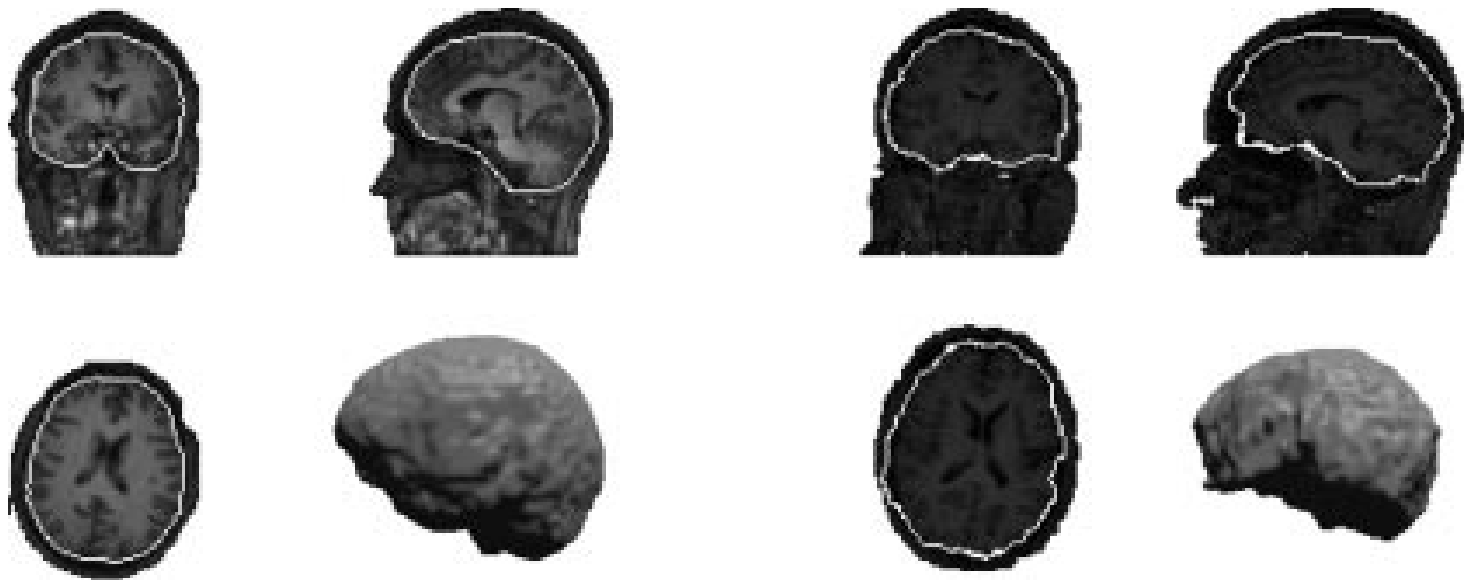

(g)
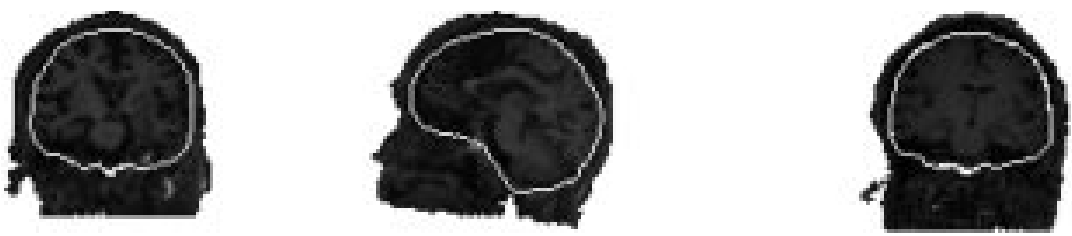

(h)
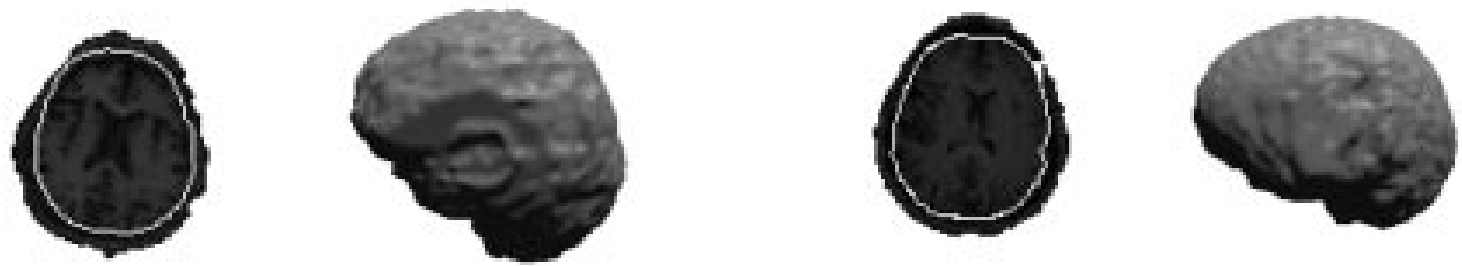

(i)

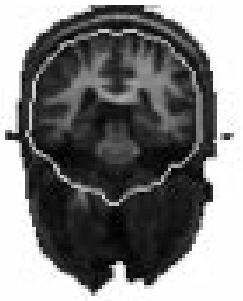

(j)
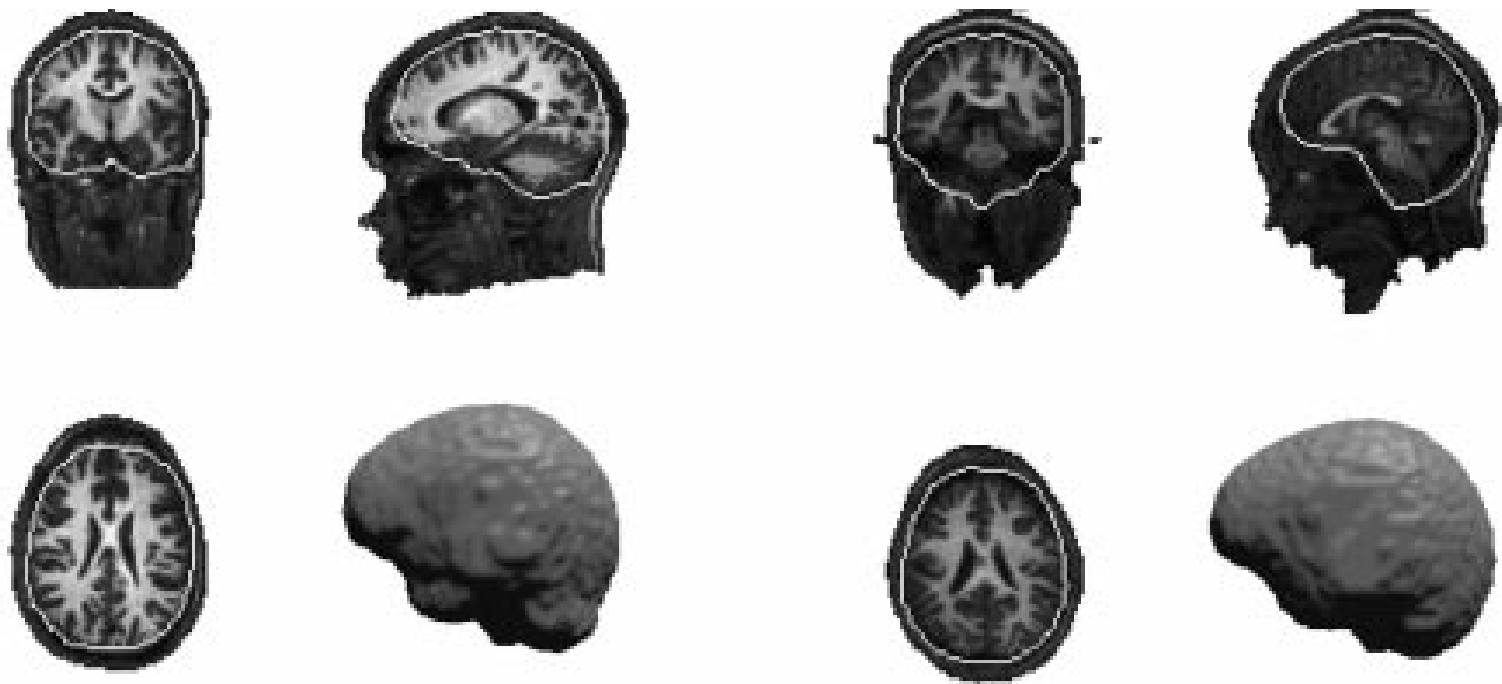

(k)

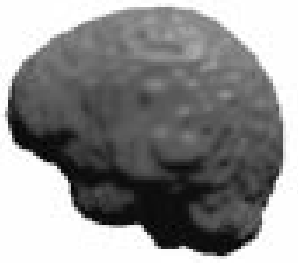

(1)

Fig. 4. (Continued.) (g)-(1) Brain isolation on 3-D MRIs The multiplanar view of the isolated brain is superimposed on the original MRI data. A 3-D rendering of the corresponding surface is also displayed.

MRI). These structures do not overlap but the deformable model represents the relative location of the head and brain contours and accounts for the anatomical variability observed among the training population. The deformable model (restricted here to head and brain surfaces) is used as a probabilistic atlas that constrains the rigid registration of the image pair.

The multimodal rigid registration method relies on the following steps: 
TABLE II

VALIDATION OF THE RESULTS ISOLATING THE BRAIN FROM 3-D HEAD MRIS By Using the Probabilistic Deformable Model. The Average AND THE STANDARD DEVIATION ARE DisPlaYED FOR THE DiFFERENT CONSIDERED QuALITY MEASURES

\begin{tabular}{c|c}
\hline & $\mu \pm \sigma$ \\
\hline \hline Sensitivity & $0.88 \pm 0.11$ \\
\hline Specificity & $0.95 \pm 0.07$ \\
\hline Accuracy & $0.96 \pm 0.05$ \\
\hline Overlap & $0.74 \pm 0.14$ \\
\hline
\end{tabular}

1) Segmentation of the head structure in MRI and the brain structure in SPECT from their backgrounds.

2) Brain isolation from the MRI using the segmentation algorithm presented in Section IV-A.

3) Registration of the estimated brain surface with the SPECT brain surface by optimization of a cost function.

The first step is standard preprocessing for background noise elimination. The second step estimates the brain surface from the MRI using the head surface parameterization and the SDM. By these means, multimodal image registration is also a measure for the accuracy of the segmentation process. Finally, the third step brings into alignment the estimated MRI brain surface and the SPECT image surface by optimization of an objective function having as variables the rigid transformation parameters between the two surfaces. Various cost functions may be used in this step for the registration of binary surfaces. We have applied the following energy function

$$
E(\Theta)=\sum_{p \in I_{\mathrm{SPECT}}} I_{D}\left(T_{\Theta}(p)\right)
$$

where

$T_{\Theta}$ rigid transformation with parameters $\Theta=$ $\left\{t_{x}, t_{y}, t_{z}, \theta_{x}, \theta_{y}, \theta_{z}\right\}$;

$p \quad$ voxel of the SPECT image surface $I_{\mathrm{SPECT}}$;

$I_{D} \quad$ chamfer distance transformation [19] of the part of the statistical model describing the brain.

For all of the SPECT surface voxels, (34) counts the distance between a SPECT image surface point and its nearest point on the deformable model surface. We have chosen chamfer distance matching because it is fast and it is easily generalized to any surfaces.

\section{Results and Validation of Registration}

Fig. 5 shows an example of a MRI/SPECT registration using the proposed technique. The images in Fig. 5(a) show the two volumes before registration. The SPECT contours are superimposed onto the MRI to qualitatively evaluate the registration. Fig. 5(b) presents the head and brain surface recovery from the MRI using the segmentation algorithm described in the previous section. The matching of the SPECT volume to the part of the model describing the brain is illustrated in Fig. 5(c). The images in Fig. 5(d) show the two volumes after registration. As can be seen, although the MRI and SPECT head and brain contours do not overlap, the two images have been correctly registered using the SDM. The whole registration procedure takes about $10 \mathrm{~min}$ cpu time on a HP C200 workstation for a $128^{3}$ image volume.

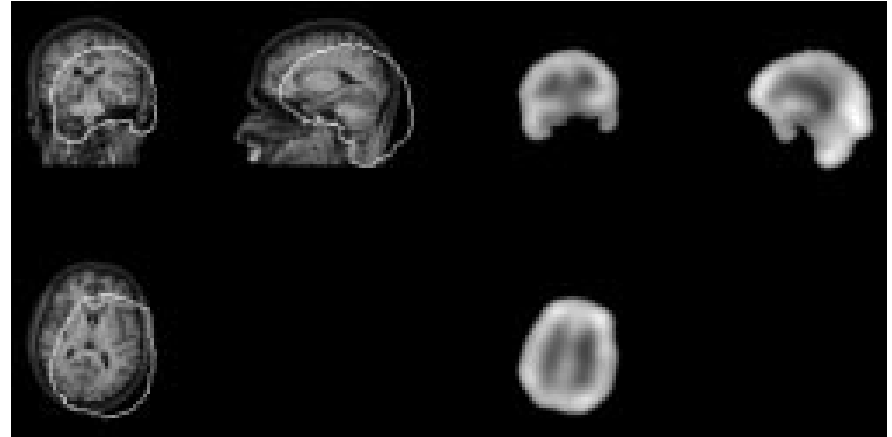

(a)

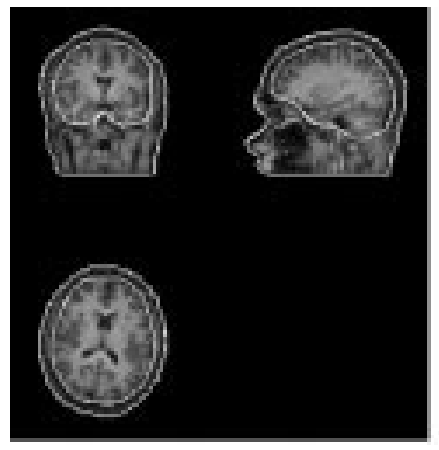

(b)

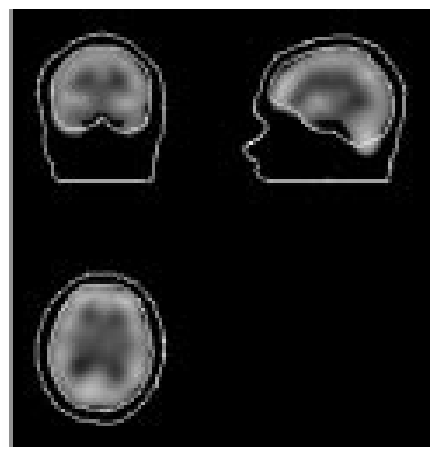

(c)

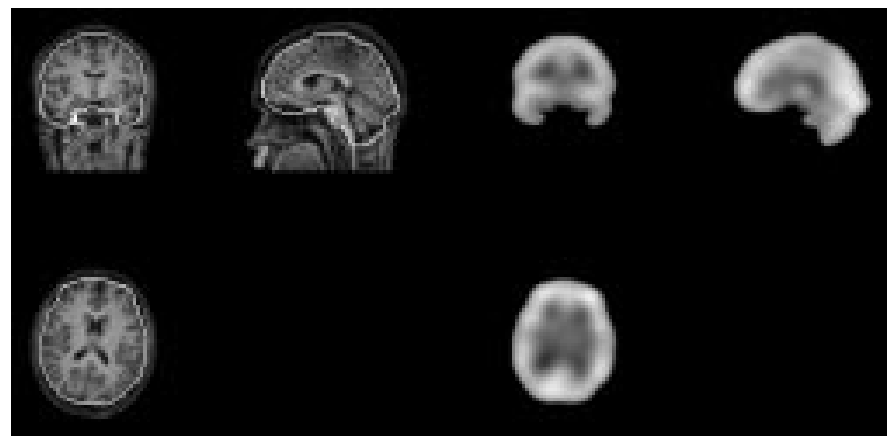

(d)

Fig. 5. MRI/SPECT registration using the deformable model. (a) MRI and SPECT volumes before registration. The SPECT contours are superimposed onto the MRI to illustrate the misalignment. (b) Parameterization of the head structure and estimation of the brain surface of the MR image in (a) using the statistically constrained deformable model. (c) Registration of the SPECT image to the part of the statistical model describing the brain surface. (d) MRI and SPECT volumes after registration. The registered SPECT image contours are superimposed onto the MRI to illustrate the alignment of the two images.

To quantitatively assess the ability of the physics-based SDM to handle multimodal image pairs, 15 SPECT image volumes were manually registered to their corresponding MRI volume (with the aid of an expert physician) to provide ground truth. One of the manually registered SPECT volumes was transformed using simulated translations between -20 and +20 voxels and rotations between -30 and +30 degrees. By these means, 25 new images were generated, yielding 25 MRI-SPECT pairs with simulated transformations and 15 original pairs with real transformations and ground truth.

The image pairs were then registered using three different techniques, and statistics on the registration errors were computed from the different registrations. We have compared our SDM-based technique with the maximization of the mutual 
TABLE III

MULTIMODAL REGISTRATION OF 3-D MRI/SPECT IMAGES. A 3-D SPECT IMAGE VOluME MANUALLY PREREGISTERED BY AN EXPERT TO ITS MRI COUNTERPART WAS ARTIFICIALLY TRANSFORMED USING 25 DIFFERENT

TRANSLATION AND Rotation Parameters. THE AVERAGE AND THE

STANDARD DEVIATION OF THE REGISTRATION ERRORS ARE PRESENTED FOR THE DiFFERENT METHODS. TRANSLATION ERRORS ARE GIVEN IN VOXELS AND

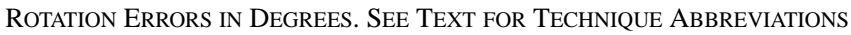

\begin{tabular}{c|c|c|c}
\hline & MI & RIU & SDM \\
\hline \hline$\Delta t_{x}$ & $1.33 \pm 1.16$ & $0.47 \pm 0.41$ & $0.89 \pm 0.43$ \\
\hline$\Delta t_{y}$ & $1.61 \pm 1.06$ & $1.13 \pm 0.90$ & $0.86 \pm 0.88$ \\
\hline$\Delta t_{z}$ & $1.06 \pm 1.19$ & $1.08 \pm 0.74$ & $1.05 \pm 1.02$ \\
\hline$\Delta \theta_{x}$ & $1.26 \pm 1.09$ & $0.75 \pm 0.56$ & $1.15 \pm 1.11$ \\
\hline$\Delta \theta_{y}$ & $1.60 \pm 0.92$ & $0.58 \pm 0.44$ & $1.28 \pm 0.87$ \\
\hline$\Delta \theta_{z}$ & $0.99 \pm 0.86$ & $1.04 \pm 0.78$ & $1.29 \pm 0.67$ \\
\hline
\end{tabular}

TABLE IV

Multimodal REgistration of 3-D MRI/SPECT IMAGES. A 3-D SPECT IMAGE VOLUME MANUALLY PREREGISTERED BY AN EXPERT TO ITS MRI

COUNTERPART WAS ARTIFICIALLY TRANSFORMED USING 25 DIFFERENT

TRANSLATION AND Rotation PARAMETERS. THE MEDIAN AND MAXIMUM REGISTRATION ERRORS FOR THE RIGID TRANSFORMATION PARAMETERS ARE PRESENTED. TRANSLATION ERRORS ARE GIVEN IN VOXELS AND RotaTION ERrors in Degrees. SeE TeXt For Technique AbBreviations

\begin{tabular}{l|c|c|c}
\hline & MI & RIU & SDM \\
\hline median $(\Delta t)$ & 1.35 & 0.63 & 0.54 \\
\hline maximum $(\Delta t)$ & 4.24 & 3.05 & 2.63 \\
\hline median $(\Delta \theta)$ & 1.14 & 0.52 & 1.09 \\
\hline maximum $(\Delta \theta)$ & 4.35 & 2.47 & 3.52 \\
\hline
\end{tabular}

TABLE $\mathrm{V}$

Multimodal REGISTRATION OF FifTEEN ORIGINAL 3-D MRI/SPECT IMAGES. THE AVERAGE AND THE STANDARD DEVIATION OF THE REGISTRATION ERRORS ARE PRESENTED FOR THE DiFFERENT METHODS. TRANSLATION ERRORS ARE GIVEN IN VOXELS AND ROTATION ERRORS IN DEGREES. SEE TEXT FOR TECHNIQUe AbBREVIATIONS

\begin{tabular}{c|c|c|c}
\hline & MI & RIU & SDM \\
\hline \hline$\Delta t_{x}$ & $2.23 \pm 1.19$ & $2.38 \pm 2.14$ & $0.89 \pm 0.75$ \\
\hline$\Delta t_{y}$ & $1.56 \pm 1.11$ & $2.84 \pm 2.82$ & $0.84 \pm 0.71$ \\
\hline$\Delta t_{z}$ & $2.47 \pm 1.52$ & $3.59 \pm 1.24$ & $1.49 \pm 0.22$ \\
\hline$\Delta \theta_{x}$ & $1.38 \pm 1.26$ & $1.59 \pm 1.24$ & $1.69 \pm 1.48$ \\
\hline$\Delta \theta_{y}$ & $1.21 \pm 1.02$ & $2.91 \pm 1.29$ & $1.85 \pm 1.60$ \\
\hline$\Delta \theta_{z}$ & $1.83 \pm 1.44$ & $2.07 \pm 1.47$ & $0.81 \pm 0.70$ \\
\hline
\end{tabular}

TABLE VI

Multimodal REGISTRATION OF FIFTEEN ORIGINAL 3-D MRI/SPECT IMAGES. THE MEDIAN AND MAXIMUM REGISTRATION ERRORS FOR THE Rigid TRANSFORMation Parameters ARE PRESENTED. TRANSLATION ERRORS ARE GIVEN IN VOXELS AND ROTATION ERRORS IN DEGREES. SeE TEXT For TeChNique AbBreviations

\begin{tabular}{l|c|c|c} 
& MI & RIU & SDM \\
\hline \hline median $(\Delta t)$ & 2.08 & 2.93 & 1.01 \\
\hline maximum $(\Delta t)$ & 4.20 & 4.73 & 1.64 \\
\hline median $(\Delta \theta)$ & 1.47 & 2.19 & 1.11 \\
\hline maximum $(\Delta \theta)$ & 3.42 & 4.29 & 3.17 \\
\hline
\end{tabular}

information (MI) [26] (currently considered as a reference method) and the robust interimage uniformity criterion (RIU), a robust statistics voxel-based method, developed by the authors [16]. Both of the latter techniques have been validated in previous studies and are robust to missing data, outliers and large rotations. For each method, the estimated registration parameters, that is the 3 -D translations $\left(t_{x}, t_{y}, t_{z}\right)$ and rotations $\left(\theta_{x}, \theta_{y}, \theta_{z}\right)$ were compared with ground truth to determine the accuracy of the registration.

Tables III and IV show the mean, standard deviation, median, and maximum of the registration errors for the different techniques applied on the 25 image pairs with simulated transformations. Tables V and VI show the same measures applied on the 15 original MRI-SPECT pairs. As can be seen, the deformable model-based approach leads to a registration accuracy which is close to or even outperforms the two other (reference) methods, both on the simulated examples and on the real cases.

\section{CONCLUSION}

We have presented a physically based 3-D SDM embedding information on the spatial relationships and anatomical variability of multiple anatomical structures, as observed over a representative training population. The model has been used to describe different brain structures (head, brain surface, ventricles, and cerebellum). Preliminary applications of the SDM included the automatic segmentation of the ICC (brain isolation) as well as the automatic registration of MRI/SPECT image pairs. Quantitative validation has shown that a 24-patients trained model was able to provide automatic accurate brain isolations and MRI/SPECT registrations on individuals not belonging to the training set.

The major advantage of statistical models is that they naturally introduce a priori statistical knowledge that provides useful constraints for ill-posed image processing tasks, such as image segmentation. Consequently, they are less affected by noise, missing data or outliers. As an example, the SDM was applied to the isolation of the brain structure from postoperative images, in which missing anatomical structures lead standard voxel-based techniques to erroneous segmentations. The registration of multimodal brain images was also handled without performing any preprocessing to remove nonbrain structures.

The SDM presented in this paper may be considered as a first step toward the development of a general purpose probabilistic anatomical atlas of the brain, for 3-D segmentation, labeling, registration and pathology characterization.

\section{REFERENCES}

[1] J. S. Duncan and N. Ayache, "Medical image analysis: Progress over two decades and the challenges ahead," IEEE Transactions on Pattern Analysis and Machine Intelligence, vol. 22, no. 1, pp. 85-106, Jan. 2000.

[2] T. McInerney and D. Terzopoulos, "Deformable models in medical image analysis: A survey," Med. Image Anal., vol. 2, no. 1, pp. 91-108, 1996.

[3] J. Martin, A. Pentland, S. Sclaroff, and R. Kikinis, "Characterization of neuropathological shape deformations," IEEE Trans. Pattern Anal. Machine Intell., vol. 20, pp. 97-112, Feb. 1998.

[4] P. Thompson, D. MacDonald, M. Mega, C. Holmes, A. Evans, and A. Toga, "Detection and mapping of abnormal brain structure with a probabilistic atlas of cortical surfaces," J. Comput. Assist. Tomogr., vol. 21, no. 4, pp. 567-581, 1997.

[5] C. Davatzikos, "Spatial normalization of 3D brain images using deformable models," J. Comput. Assist. Tomogr., vol. 20, no. 4, pp. 656-665, 1996.

[6] J. Gee, M. Reivich, and R. Bajcsy, "Elastically deforming 3D atlas to match anatomical brain images," J. Comput. Assist. Tomogr., vol. 17, no. 2, pp. 225-236, 1993.

[7] N. Duta and M. Sonka, "Segmentation and interpretation of MR images of the human brain," IEEE Trans. Med. Imag., vol. 17, pp. 1049-1062, June 1999. 
[8] A. Kelemen, G. Szekely, and G. Gerig, "Elastic model-based segmentation of 3-D neuroradiological data sets," IEEE Trans. Med. Imag., vol. 18, pp. 828-839, Oct. 1999

[9] G. Székely, A. Kelemen, A. Brechbuhler, and G. Gerig, "Segmentation of $2 \mathrm{D}$ and $3 \mathrm{D}$ objects from MRI data using constrained elastic deformations of flexible Fourier contour and surface models," Med. Image Anal., vol. 1, no. 1, pp. 19-34, 1996.

[10] C. Nastar and N. Ayache, "Frequency-based nonrigid motion analysis: Application to four-dimensional medical images," IEEE Trans. Pattern Anal. Machine Intell., vol. 18, pp. 1069-1079, Nov. 1996.

[11] A. Pentland and S. Sclaroff, "Closed-form solutions for physically based shape modeling and recognition," IEEE Trans. Pattern Anal. Machine Intell., vol. 13, pp. 730-742, July 1991

[12] T. F. Cootes, C. J. Taylor, and J. Graham, "Active shape models-Their training and application," Comput. Vis. Image Understanding, vol. 1, no. 1, pp. 38-59, 1995

[13] A. D. Brett and C. J. Taylor, "A framework for automated landmark generation for automated 3D statistical model construction," in Proc. 16th Int. Conf. Information Processing in Medical Imaging, Visegrad, Hungary, June 1999, pp. 376-381.

[14] - "Construction of 3D shape models of femoral articular cartilage using harmonic maps," in Proc. Medical Image Computing and Computer Assisted Intervention (MICCAI), Pittsburgh, PA, Oct. 11-14, 2000, pp. $1205-1214$.

[15] C. Nastar, B. Moghaddam, and A. Pentland, "Flexible images: Matching and recognition using learned deformations," Comput. Vis. Image Understanding, vol. 65, no. 2, pp. 179-191, 1997.

[16] C. Nikou, J. P. Armspach, F. Heitz, I. J. Namer, and D. Grucker, "MR/MR and MR/SPECT registration of brain images by fast stochastic optimization of robust voxel similarity measures," Neuroimage, vol. 8 , no. 1 , pp. $30-43,1998$.
[17] A. Kotcheff and C. Taylor, "Automatic construction of eigenshape models by genetic algorithms," in Information Processing in Medical Imaging. Berlin, Germany: Springer-Verlag, 1997, pp. 1-14.

[18] G. Bueno, O. Musse, F. Heitz, and J. P. Armspach, "3D segmentation of anatomical structures in MR images on large data sets," Magn. Reson. Imag., vol. 19, pp. 73-88, 2001.

[19] G. Borgefors, "On digital distance tranforms in three dimensions," Comput. Vis. Image Understanding, vol. 64, no. 3, pp. 368-376, 1996

[20] K. J. Bathe, Finite Element Procedures. Englewood Cliffs, NJ: Prentice-Hall, 1996.

[21] C. Kervrann and F. Heitz, "A hierarchical Markov modeling approach for the segmentation and tracking of deformable shapes," Graphical Models Image Processing, vol. 60, no. 3, pp. 173-195, 1998

[22] M. Kass, A. Witkin, and D. Terzopoulos, "Snakes: Active contour models," Int. J. Comput. Vis., vol. 4, no. 1, pp. 321-331, 1988.

[23] J. Declerck, G. Subsol, J. P. Thirion, and N. Ayache, "Automatic retrieval of anatomical structures in 3-D medical images," INRIA, France, Tech Rep. 2485, Feb. 1995.

[24] C. E. Metz, "Basic principles of ROC analysis," Semin. Nucl. Med., vol. 8, pp. 283-298, 1978

[25] X. Zeng, L. Staib, R. Schultz, and J. Duncan, "Segmentation and measurement of the cortex from 3-D MR images using coupled-surfaces propagation," IEEE Trans. Med. Imag., vol. 18, pp. 927-937, Oct. 1999.

[26] F. Maes, A. Collignon, D. Vandermeulen, G. Marchal, and P. Suetens, "Multimodality image registration by maximization of mutual information,” IEEE Trans. Med. Imag., vol. 16, pp. 187-198, Apr. 1997. 